\title{
INEQUALITIES CONCERNING THE INVERSES OF POSITIVE DEFINITE MATRICES
}

\author{
by W. W. MUIR \\ (Received 4th October 1972)
}

\section{Introduction}

Much has been written on inequalities concerning positive definite matrices, but a new insight may be gained by examining inequalities from the standpoint of the inverse matrix. The standard inequality of Hölder can then be used in a more fruitful manner. This leads to some new results and a rediscovery of some known results.

\section{Compound matrices}

The following theory requires the use of the Binet-Cauchy theory of compound matrices which is described here. $\quad M$ is a given $m \times n$ matrix and $k$ is an integer less than the smaller of $m$ and $n . \alpha$ is a subset of $k$ integers from the set $(1,2, \ldots, m)$ and $\beta$ is a subset of $k$ integers from the set $(1,2, \ldots, n)$. Suppose we delete all rows of $M$ whose indices do not belong to $\alpha$ and also all columns whose indices do not belong to $\beta$. The determinant of the remaining $k \times k$ matrix is denoted by $[M]_{\alpha \beta}$ or $m_{\alpha \beta}$. The matrix whose elements are $m_{\alpha \beta}$ is denoted by $M^{(k)}$. The priority of the elements in rows or columns is in lexicographical order of the elements of either the set $\alpha$ or the set $\beta$ respectively. $M^{(k)}$ therefore is a matrix of order $m_{(k)} \times n_{(k)}$ where $m_{(k)}=m ! /(k !(m-k) !)$. It can be proved (Aitken (1), p. 94) that

$$
(M N)^{(k)}=M^{(k)} N^{(k)} \text {. }
$$

\section{The inverse log-convex property}

In the text that follows it is assumed that $A$ and $B$ are positive definite real symmetric matrices each of order $n \times n$ and $\lambda$ and $\mu$ are real non-negative numbers such that $\lambda+\mu=1$.

Let $f(M)$ be a scalar function of the elements of a matrix $M$. Then if

$$
f\left((\lambda A+\mu B)^{-1}\right) \leqq\left\{f\left(A^{-1}\right)\right\}^{\lambda}\left\{f\left(B^{-1}\right)\right\}^{\mu} \leqq \lambda f\left(A^{-1}\right)+\mu f\left(B^{-1}\right)
$$

we say that the function $f$ possesses the inverse logconvex property or ILC property for short. We note that if $f\left(A^{-1}\right)$ and $f\left(B^{-1}\right)$ are real non-negative numbers then the right-hand inequality follows (Bellman (2), p. 129).

\section{A basic theorem}

Let $X$ be any real matrix and let $g(M)=\left[X^{\prime} M X\right]_{\alpha x}$. Then the function $g$ has the ILC property. 
Since $A$ and $B$ are positive definite real symmetric matrices of order $n \times n$, we can find a matrix $P$ such that

and

$$
P^{\prime} A P=I, P^{\prime} B P=F=\operatorname{diag}\left(\gamma_{1}, \gamma_{2}, \ldots, \gamma_{n}\right)
$$

It follows that

$$
\gamma_{i}>0 \text { for all } i \text {. }
$$

$$
(\lambda A+\mu B)^{-1}=P(\lambda I+\mu F)^{-1} P^{\prime}
$$

We use also two inequalities:

(i) If $x$ and $y$ are real positive or zero numbers then (Bellman (3), p. 129),

$$
\lambda x+\mu y \geqq x^{\lambda} y^{\mu}
$$

(ii) If $\mathbf{u}_{i}$ and $v_{i}$ are non-negative for $i=1,2, \ldots, n$ then (Hölder's Inequality)

$$
\sum_{i=1}^{n} u_{i}^{\lambda} v_{i}^{\mu} \leqq\left(\sum_{i=1}^{n} u_{i}\right)^{\lambda}\left(\sum_{i=1}^{n} v_{i}\right)^{\mu}
$$

From (1.1) and (3.1) it follows that

$$
\left(P^{\prime}\right)^{(k)} A^{(k)} P^{(k)}=I \text { and } P^{\prime(k)} B^{(k)} P^{(k)}=F^{(k)},
$$

where for instance if $k=3$, from the matrix $F^{(k)}$ we obtain

Now

$$
f_{11}=\gamma_{1} \gamma_{2} \gamma_{3}, f_{22}=\gamma_{1} \gamma_{2} \gamma_{4}, \ldots, \text { etc. }
$$

$$
\begin{aligned}
{\left[X^{\prime}(\lambda A+\mu B)^{-1} X\right]_{\alpha \alpha} } & =\left[X^{\prime} P(\lambda I+\mu F)^{-1} P^{\prime} X\right]_{\alpha \alpha} \\
& =\left[U^{\prime}(\lambda I+\mu F)^{-1} U\right]_{\alpha \alpha}\left(\text { where } U=P^{\prime} X\right) \\
& =\sum_{\beta} \sum_{\gamma} u_{\alpha \beta}^{\prime}\left[(\lambda I+\mu F)^{-1}\right]_{\beta \gamma} u_{\gamma \alpha} \\
& =\sum_{\beta} u_{\alpha \beta}^{\prime}\left[(\lambda I+\mu F)^{-1}\right]_{\beta \beta} u_{\beta \alpha} \text { (as } \lambda I+\mu F \text { is diagonal) } \\
& =\sum_{\beta}\left\{\frac{u_{\beta \alpha}^{2}}{\left(\lambda+\mu \gamma_{i}\right)\left(\lambda+\mu \gamma_{j}\right) \ldots\left(\lambda+\mu \gamma_{l}\right)}\right\}
\end{aligned}
$$

and $\beta$ represents the subset $(i, j, \ldots, l)$ from the numbers $(1,2, \ldots, n)$. From (3.2) therefore

$$
\begin{aligned}
{\left[X^{\prime}(\lambda A+\mu B)^{-1} X\right]_{\alpha \alpha} } & \leqq \sum_{\beta}\left\{\frac{u_{\beta \alpha}^{2}}{\left(\gamma_{i} \gamma_{j} \ldots \gamma_{l}\right)^{\mu}}\right\} \\
& =\sum_{\beta} u_{\beta \alpha}^{2 \lambda}\left\{\frac{\left.u_{\beta \alpha}^{2}\right\}^{\mu}}{f_{\beta \beta}}\right\} \\
& \leqq\left\{\sum_{\beta} u_{\beta \alpha}^{2}\right\}^{\lambda}\left\{\sum_{\beta} \frac{u_{\beta \alpha}^{2}}{f_{\beta \beta}}\right\}^{\mu}
\end{aligned}
$$

by Hölder's inequality. 
By choosing appropriate values of $\lambda$ and $\mu$ in (3.4) we obtain

$$
\left[X^{\prime} A^{-1} X\right]_{x \alpha}=\sum_{\beta} u_{\beta \alpha}^{2} ;\left[X^{\prime} B^{-1} X\right]_{\alpha \alpha}=\sum_{\beta}\left(\frac{u_{\beta \alpha}^{2}}{f_{\beta \beta}}\right) \text {. }
$$

Hence (3.5) gives

$$
\left[X^{\prime}(\lambda A+\mu B)^{-1} X\right]_{\alpha \alpha} \leqq\left\{\left[X^{\prime} A^{-1} X\right]_{x x}\right\}^{\lambda}\left\{\left[X^{\prime} B^{-1} X\right]_{x x}\right\}^{\mu}
$$

and the result is proved.

\section{Deductions from the basic theorem}

(a) It follows that $g(M)=[M]_{x \alpha}$ is a matrix function that has the ILC property:

in particular if $k=1$ then

$$
\left[(\lambda A+\mu B)^{-1}\right]_{i i} \leqq\left\{\left[A^{-1}\right]_{i i}\right\}^{\lambda}\left\{\left[B^{-1}\right]_{i i}\right\}^{\mu},
$$

which is given as Bergström's Inequality in Bellman (2, p. 131). Also if $k=n$ we obtain

(Bellman (2), p. 128).

$$
|\lambda A+\mu B| \geqq|A|^{\lambda}|B|^{\mu}
$$

(b) Let $\phi_{k}(M)=\sum_{\alpha} m_{\alpha \alpha}$. Thus $\phi_{k}(M)$ is the sum of the products of the eigenvalues of $M$ taken $k$ at a time.

Then $\phi_{k}$ is a function with the ILC property.

Proof.

$$
\begin{aligned}
\phi_{k}\left((\lambda A+\mu B)^{-1}\right) & =\sum_{\alpha}\left[(\lambda A+\mu B)^{-1}\right]_{\alpha \alpha} \\
& \leqq \sum_{\alpha}\left\{\left[A^{-1}\right]_{\alpha \alpha}\right\}^{\lambda}\left\{\left[B^{-1}\right]_{\alpha \alpha}\right\}^{\mu} \\
& \leqq\left\{\sum_{\alpha}\left[A^{-1}\right]_{\alpha \alpha}\right\}^{\lambda}\left\{\sum_{\alpha}\left[B^{-1}\right]_{\alpha \alpha}\right\}^{\mu} \\
& =\left\{\phi_{k}\left(A^{-1}\right)\right\}^{\lambda}\left\{\phi_{k}\left(B^{-1}\right)\right\}^{\mu}
\end{aligned}
$$

If $k=1$ then (4.2) yields

$$
\operatorname{tr}\left\{(\lambda A+\mu B)^{-1}\right\} \leqq\left\{\operatorname{tr}\left(A^{-1}\right)\right\}^{\lambda}\left\{\operatorname{tr}\left(B^{-1}\right)\right\}^{\mu},
$$

where tr stands for trace. Hence the trace of a matrix possesses the ILC property.

(c) Let $M$ be a positive definite symmetric matrix and let the eigenvalues be

$$
l_{1}, l_{2}, \ldots, l_{n} \text {, where } l_{1} \geqq l_{2} \geqq \ldots, \geqq l_{n}>0 \text {. }
$$

Let $L_{k}(M)=l_{1} l_{2} \ldots l_{k}$.

Then $L_{k}$ is a function that follows the ILC property.

The proof from the basic theorem is omitted since this result has already been given in equivalent form in Bellman (2, p. 130). 


\section{Certain types of matrices $A$ and $B$}

Suppose we partition $A$ as

$$
A=\left[\begin{array}{ll}
A_{11} & A_{12} \\
A_{21} & A_{22}
\end{array}\right]
$$

where $A_{11}$ is of order $p \times p$ say. Let

Let $\lambda=\mu=\frac{1}{2}$ and hence

$$
B=\left[\begin{array}{rr}
A_{11} & -A_{12} \\
-A_{21} & A_{22}
\end{array}\right] .
$$

$$
\lambda A+\mu B=\left[\begin{array}{cc}
A_{11} & 0 \\
0 & A_{22}
\end{array}\right] .
$$

$A, B, \lambda$ and $\mu$ are defined as above from now on in this paper.

Let $D=A^{-1}$ and let $D$ be partitioned similarly to $A$ with

Then it is easy to prove that

$$
D=\left[\begin{array}{ll}
D_{11} & D_{12} \\
D_{21} & D_{22}
\end{array}\right]
$$

$$
B^{-1}=\left[\begin{array}{rr}
D_{11} & -D_{12} \\
-D_{21} & D_{22}
\end{array}\right]
$$

and that the eigenvalues of $A$ and $B$ are the same.

The determinant of a principal submatrix of $A$ is the same as the determinant of the corresponding principal submatrix of $B$ and the same applies to their inverses. In our previous notation therefore we obtain

Hence from (4.1)

$$
\left[A^{-1}\right]_{\alpha \alpha}=\left[B^{-1}\right]_{\alpha x} \text {. }
$$

$$
\left[(\lambda A+\mu B)^{-1}\right]_{\alpha \alpha} \leqq\left[A^{-1}\right]_{\alpha \alpha}^{\lambda}\left[B^{-1}\right]_{\alpha \alpha}^{\mu}
$$

and inserting the appropriate values of $\lambda, \mu, A$ and $B$ and letting $\alpha=1,2, \ldots, p$ we obtain

$$
\left|A_{11}^{-1}\right| \leqq\left|D_{11}\right|
$$

i.e. $\left|D_{11}\right| \geqq 1 /\left|A_{11}\right|$ (De Bruijn (3), page 28.)

\section{Further inequalities concerning products of largest eigenvalues}

Following the definition $L_{k}$ of the product of largest eigenvalues and using (4.3) and (5.1) we obtain

$$
L_{k}\left(A^{-1}\right) \geqq L_{k}^{-}\left[\begin{array}{ll}
A_{11}^{-1} & 0 \\
0 & A_{22}^{-1}
\end{array}\right] .
$$

Thus $L_{k}\left(A^{-1}\right) \geqq L_{k}\left(A_{11}^{-1}\right)$, or, if we take $r$ of the eigenvalues from $A_{11}^{-1}$ and the rest from $A_{22}^{-1}$, we obtain

$$
L_{k}\left(A^{-1}\right) \geqq L_{r}\left(A_{11}^{-1}\right) \cdot L_{k-r}\left(A_{22}^{-1}\right) .
$$


Suppose the eigenvalues of $A$ are $\alpha_{1} \geqq \alpha_{2} \geqq \alpha_{3} \geqq \ldots \geqq \alpha_{n}>0$; then it follows from (6.1) that

$$
\frac{1}{\alpha_{n}} \cdot \frac{1}{\alpha_{n-1}} \ldots \frac{1}{\alpha_{n-k+1}} \geqq \frac{1}{a_{11}} \frac{1}{a_{22}} \ldots \frac{1}{a_{k k}}
$$

or $\alpha_{n} \alpha_{n-1} \ldots \alpha_{n-k+1} \leqq a_{11} a_{22} \ldots a_{k k}$. (See Bellman (2), page 134.)

\section{Further inequalities concerning sums of products of eigenvalues}

As $\phi_{k}(A)$ is the sum of principal minors of order $k$ and using the definitions of $A$ and $B$ given in Section 5 and the relationship (5.2) we see that

Hence from (4.2)

$$
\phi_{k}\left(A^{-1}\right)=\phi_{k}\left(B^{-1}\right) \text {. }
$$

$$
\begin{aligned}
& \begin{array}{l}
\phi_{k}\left(A^{-1}\right) \geqq \phi_{k}\left[\begin{array}{ll}
A_{11}^{-1} & 0 \\
0 & A_{22}^{-1}
\end{array}\right] \\
\qquad \phi_{k}\left(A_{11}^{-1}\right)+\phi_{k-1}\left(A_{11}^{-1}\right) \phi_{1}\left(A_{22}^{-1}\right)+\phi_{k-2}\left(A_{11}^{-1}\right) \phi_{2}\left(A_{22}^{-1}\right)
\end{array} \\
& \text { or in short, if } \phi_{0}(A)=1 \text {, then } \quad+\ldots+\phi_{1}\left(A_{11}^{-1}\right) \phi_{k-1}\left(A_{22}^{-1}\right)+\phi_{k}\left(A_{22}^{-1}\right)
\end{aligned}
$$

$$
\phi_{k}\left(A^{-1}\right) \geqq \sum_{\mathrm{r}=0}^{k} \phi_{r}\left(A_{11}^{-1}\right) \phi_{k-r}\left(A_{22}^{-1}\right) .
$$

If $k=n$ then from (7.1)

$$
|A| \leqq\left|A_{11}\right|\left|A_{22}\right|
$$

the well-known Hadamard-Fischer theorem.

If $k=1$, from (7.1) it follows that

$$
\operatorname{tr}\left(A^{-1}\right) \geqq \operatorname{tr}\left(A_{11}^{-1}\right)+\operatorname{tr}\left(A_{22}^{-1}\right),
$$

a sort of dual to the Hadamard-Fischer theorem.

Also we see from (5.3) and (7.2) that

$$
\phi_{k}\left(A^{-1}\right)=\sum_{\alpha}\left[A^{-1}\right]_{\alpha \alpha} \geqq \sum_{\alpha} \frac{1}{[A]_{\alpha \alpha}} \geqq \sum \frac{1}{a_{11} a_{22} \ldots a_{k k}} .
$$

\section{REFERENCES}

(1) A. C. Artken, Determinants and Matrices (Oliver and Boyd, 1964).

(2) R. Bellman, Introduction to Matrix Analysis, 2nd Edition (McGraw-Hill, 1970).

(3) N. G. DE BRUIJN, Inequalities concerning minors and eigenvalues, Nieuw Arch. Wisk. 3 (1956), 18-35. 